\title{
Knowledge and behaviors of drunk-driving offenders in Guangzhou, China
}

\author{
Keqin Jia ${ }^{1}$, Judy Fleiter ${ }^{1}$, Mark King ${ }^{1}$, Mary Sheehan ${ }^{1}$, Wenjun $\mathrm{Ma}^{2}$, and Jianzhen Zhang ${ }^{3}$ \\ ${ }^{1}$ The Centre for Accident Research and Road Safety—Queensland, Queensland University of Technology, Australia \\ ${ }^{2}$ Guangdong Provincial Institute of Public Health, Center for Disease Control and Prevention of Guangdong Province, China \\ ${ }^{3}$ School of Medicine, University of Queensland, Australia
}

\begin{abstract}
Aims: To better understand the knowledge and behaviors of drunk-driving offenders relating to alcohol use and driving in the context of recently amended Chinese legislation, and to investigate the involvement of alcohol-use disorders.

Design: The study was a cross-sectional survey conducted in 2012.

Setting and participants: Data were collected at a local jail and 101 participants were recruited while in detention.

Measures: Questionnaire items examined demographic characteristics as well as practices and knowledge relating to alcohol use and driving. The Alcohol Use Disorders Identification Test (AUDIT) was used to assess hazardous drinking levels.

Findings: Knowledge about the two legal limits for "drink driving” and for "drunk driving” was low, at $28.3 \%$ and $41.4 \%$, respectively. AUDIT scores indicated that a substantial proportion of the offenders had high levels of alcohol-use disorders. Higher AUDIT scores were found among the least experienced drivers, those who lacked knowledge about the legal limits, and recidivist drunk drivers.

Conclusions: Limited awareness of legal alcohol limits might contribute to offending; high AUDIT scores suggest that hazardous drinking levels may also contribute. This study provides important information to assist in refining community education and prevention efforts.
\end{abstract}

\section{Introduction}

Alcohol is a major contributor to road crashes in China (Cochrane, Chen, Conigrave, \& Hao, 2003; Li, Xie, Nie, \& Zhang, 2012). According to statistics from the Traffic Management Bureau under the Ministry of Public Security, in 2011 there were 4,912 traffic crashes related to drink driving in China, resulting in 1,957 deaths and 5,221 serious injuries (2012). Two levels of offense are defined in legislation: "drink driving" is defined as a blood alcohol concentration (BAC) $20 \mathrm{mg} / 100 \mathrm{ml} \leq \mathrm{BAC}<80 \mathrm{mg} / 100 \mathrm{ml}$, and "drunk driving" is defined as a BAC $\geq 80 \mathrm{mg} / 100 \mathrm{ml}$. The legislative context of drunk driving has changed in recent years with the introduction of a legislative amendment to make drunk driving a criminal offense on May 1, 2011. The penalties for drunk driving include disqualification of license for five years, one to six months' detention, and a monetary fine. However, it is not clear whether BAC levels and the recent legislative amendments are known and/or understood by offenders.
Guangzhou is the capital of Guangdong Province in the south of China. Guangdong is China's leading province in terms of economic development and motorization (Ma, 2012). Road-crash-related injuries and fatalities have also increased, and Guangdong Province records the highest number of traffic-related injuries and fatalities among all 31 provinces in China (Traffic Management Bureau under the Ministry of Public Security, 2012; Zhang, Yau, \& Chen, 2013). In 2011, Guangzhou had 12.78 million people and 2.145 million vehicles, and the city experienced 2,664 traffic crashes resulting in 930 fatalities and 3,017 injuries. That year, 7,274 alcohol-related driving offenders were apprehended, including 1,644 drunk-driving offenders (Traffic Management Bureau under the Ministry of Public Security, 2012). According to the official statistics from the Guangzhou Public Security Bureau (Kong, 2012), from May 1, 2011, to April 30, 2012 (the first full year following the amendment that made drunk driving a criminal offense), traffic-police officers identified 4,750 drink-

Correspondence: Keqin Jia, 3 Noble Street, Bridgeman Downs, QLD 4035, Australia. Telephone: +61 7 31380526; FAX : +61 7 31384907; Email: keqin6020@gmail.com

Financial support: This research was funded by a 2012 Prime Minister's Australia Asia Endeavour Postgraduate Award (Outgoing) and an Australian Postgraduate Award.

Declaration of interest: There are no interests to declare for this research.

Keywords: drink driving, drunk driving, offender, China, AUDIT 
driving cases, among which 877 were drunk-driving offenses.

There has been very little research conducted in China since major penalty changes for alcohol-related driving were introduced in May 2011. Research conducted in other countries has identified that knowledge of safe drinking levels and how to manage alcohol consumption to remain within legal BAC limits, as well as perceptions about drinkdriving enforcement, can influence decisions about drinkdriving behavior (Baum, 2000; Tran et al., 2011; Watson \& Freeman, 2007). Therefore, the present study explored whether convicted drunk drivers were aware of the amended legislation and related BAC limits, and whether this knowledge, exposure to enforcement, and the existence of alcohol-use disorders were associated with drunk driving.

\section{Method}

\section{Participants and Procedure}

With the assistance of the Guangdong Provincial Institute of Public Health, Center for Disease Control and Prevention of Guangdong Province, and the local jail authority, 101 drunk-driving offenders in detention due to a drunk-driving conviction were recruited in one detention facility in Guangzhou. According to data published on a Guangzhou government website (Wen, 2012), 4,930 alcohol-related driving offenders were apprehended between January 1 and September 10, 2012, with 1,079 of these being drunk-driving offenders. Recruitment for the current study spanned a 6-month period (May to October, 2012) that largely overlaps with the former time period. Therefore, the current sample of 101 offenders recruited from one detention facility represents approximately $10 \%$ of the total number reported to have been apprehended in the 10-month period. A self-report questionnaire about drink-driving knowledge and practices was completed by offenders, and two trained, uniformed police officers from the jail distributed and collected completed questionnaires; the research team was prohibited from direct access to offenders in detention. The study was approved by the Queensland University of Technology's Human Research Ethics Committee.

\section{Measures}

Demographic characteristics and offender knowledge and practices in relation to drunk driving were collected. Questions covered topics such as whether participants knew that drunk driving had become a criminal offense and their knowledge of the two legal BAC limits. In addition, items assessing knowledge of alcohol metabolism were used. These questions were based on an education manual titled "Driving for Life" (Australian Drug Foundation, 2002) and Baum's study (2000) comparing the attitudes and knowledge of drink-driving offenders and the general community.

The presence of alcohol-use disorders was assessed using the Alcohol Use Disorders Identification Test (AUDIT)
(Babor \& Grant, 1989). The degree to which drinking levels were hazardous was assessed using the World Health Organization (WHO) cut-off scores (0-7.9: Low; 8-15.9: Medium; 16-19.9: High; 20+: Need further diagnostic evaluation for alcohol dependence). Because the AUDIT tool uses the concept of a "standard unit of alcohol" as the basis for calculating alcohol consumption, a concept that does not exist in China, we asked participants how much alcohol they consumed using local measurements (e.g., a Liang is approximately $50 \mathrm{ml}$ ) and local alcohol content of common beverages, which the first author then converted to standard units in order to calculate the AUDIT score. Alcohol-related driving behaviors and whether participants had previously been penalized for drink and drunk driving were also assessed.

\section{Analytical strategy}

Means for continuous variables were compared using parametric $t$ tests and one-way analysis of variance (ANOVA) and post hoc tests. Bivariate correlations between continuous variables utilized Pearson's productmoment correlation $(r)$. All analyses were evaluated at a significance level of $\alpha=0.05$. A multiple regression analysis was used to explore the relationship between AUDIT scores and other independent variables: age, gender, years as a licensed driver, age at which participants started drinking, marital status, monthly income, education level, knowledge of law and the two legal BAC levels, and recidivist status. Analyses were conducted using Statistical Package for the Social Sciences (SPSS) version 21.0.

\section{Results}

\section{Socio-demographic Characteristics}

The sample was predominantly male (90.1\%) with an average age of 33.6 years ( $S D=8.7$; range $17-59$ years). One quarter (25\%) were aged between 17 and 26 years; $50 \%$ were under 33 years; and $75 \%$ were under the age of 40. Approximately two thirds of the sample (63.4\%) was married and one third had tertiary qualifications (33\%). When asked about the frequency of alcohol consumption, results revealed that $10 \%$ reported drinking once per month or less, 38\% reported 2-4 times monthly, 33\% reported 2-3 times weekly, and $19 \%$ reported more than 4 times weekly. The majority $(73.7 \%)$ of offenders had a monthly income of more than 2,000 Renminbi (RMB); 26.7\% earned 2,000 RMB or less ( 1 US Dollar $=6.86$ RMB ). Table 1 gives a clear indication of the distribution of reported incomes. In Guangzhou, in 2011, the lowest monthly income for employees was 1,300 RMB and the average monthly income was 4,800 RMB, according to Guangdong Statistics Bureau (2012).

Just over half (53.5\%) the sample had a permanent job, while $16.7 \%$ were unemployed (including students and retired people). The number of years as a licensed driver varied from 0.5 to 27 years with an average of 8.4 years $(S D=6.8) ; 7.9 \%$ of offenders reported having no license at the time of offense. Professional drivers (e.g., taxi, bus, and truck drivers) made up $20 \%$ of the sample. 
Table 1

One-way analysis of variance (ANOVA) and post hoc tests between demographic variables and alcohol use disorders identification test score

\begin{tabular}{|c|c|c|c|c|c|c|}
\hline \multirow[b]{2}{*}{ Variable } & \multirow{2}{*}{$\begin{array}{c}\text { Number } \\
(\%)\end{array}$} & \multirow{2}{*}{$\begin{array}{l}\text { AUDIT } \\
\text { score }\end{array}$} & \multirow{2}{*}{$\begin{array}{c}\text { Mean } \\
\text { difference }\end{array}$} & \multicolumn{2}{|c|}{ 95\% Confidence Interval } & \multirow[b]{2}{*}{$P$ value } \\
\hline & & & & Lower & Upper & \\
\hline \multicolumn{7}{|l|}{ Monthly Income (RMB) } \\
\hline 0-2000 & $27(26.7)$ & 11.35 & 2.598 & -2.63 & 7.82 & 0.698 \\
\hline $2001-4000$ & 33(32.7) & 10.42 & 3.520 & -1.47 & 8.51 & 0.322 \\
\hline $4001-6000$ & 10(9.9) & 9.60 & 4.344 & -2.38 & 11.06 & 0.420 \\
\hline $6001-8000$ & $7(6.9)$ & 9.71 & 4.230 & -3.36 & 11.82 & 0.586 \\
\hline $8001-10,000$ & $5(5.0)$ & 10.40 & 3.544 & -5.07 & 12.16 & 0.837 \\
\hline More than 10,000 & 18(17.8) & 13.94 & 0 & & & \\
\hline Not stated & $1(1.0)$ & & & & & \\
\hline \multicolumn{7}{|l|}{$\begin{array}{l}\text { Alcohol Problem Severity (AUDIT } \\
\text { Score) }\end{array}$} \\
\hline Low (0-7.9) (Group 1) & $35(35)$ & 5.83 & 0 & & & \\
\hline Medium (8-15.9) (Group 2) & $45(45)$ & 10.89 & -5.060 & -6.19 & -3.93 & $<0.001^{* *}$ \\
\hline High (16-19.9) (Group 3) & $7(7)$ & 17.57 & -11.743 & -13.83 & -9.66 & $<0.001^{* *}$ \\
\hline $\begin{array}{l}\text { (Need further diagnostic evaluation for } \\
\text { alcohol dependence ( } 20 \text { or more) } \\
\text { (Group 4) }\end{array}$ & $4(4)$ & 20.00 & -14.171 & -16.83 & -11.52 & $<0.001^{* *}$ \\
\hline Medium vs & $45(45)$ & 10.89 & & & & $<0.001^{* *}$ \\
\hline High (Group 2-3) & $7(7)$ & 17.57 & -6.683 & -8.73 & -4.64 & \\
\hline Medium vs & $45(45)$ & 10.89 & & & & $<0.001^{* *}$ \\
\hline the Highest (Group 2-4) & $4(4)$ & 20.00 & -9.111 & -11.74 & -6.49 & \\
\hline High vs & $7(7)$ & 17.57 & & & & \\
\hline the Highest (Group 3-4) & $4(4)$ & 20.00 & -2.429 & -5.58 & 0.73 & 0.19 \\
\hline \multicolumn{7}{|l|}{ Education and AUDIT Score } \\
\hline 1. Primary School & 11(10.9) & 12.9 & -1.879 & -7.25 & 3.49 & 0.797 \\
\hline 2. Middle School & $22(21.8)$ & 11.8 & -0.788 & -5.03 & 3.46 & 0.962 \\
\hline 3. High School and Middle College & $34(33.7)$ & 10.2 & 0.854 & -2.91 & 4.62 & 0.934 \\
\hline 4. Bachelor degree and Higher & $33(32.6)$ & 11.0 & 0 & & & \\
\hline Missing & $1(1)$ & & & & & \\
\hline \multicolumn{7}{|l|}{ Years Licensed and AUDIT Score } \\
\hline 1. Novice driver ( $<2$ years) & 13(12.9) & 16.4 & 0 & & & \\
\hline 2. Licensed $2-5$ years & $26(25.8)$ & 11.3 & 5.115 & 0.42 & 9.82 & $0.027 *$ \\
\hline 3. Licensed 6-10 years & $30(29.7)$ & 9.9 & 6.451 & 1.86 & 11.05 & $0.002 *$ \\
\hline 4. Licensed 11 years or more & $27(26.7)$ & 8.7 & 7.654 & 2.95 & 12.35 & $<0.001^{* *}$ \\
\hline Missing & $5(5)$ & & & & & \\
\hline
\end{tabular}

$* p<0.05 ; * * p<0.001$

\section{Knowledge about BAC limits}

The majority of participants (84.2\%) knew that drunk driving had become a criminal offense in May 2011; however, their knowledge about legal BAC limits for drink driving (28.3\%) and drunk driving (41.4\%) was surprisingly low. In both cases, the most common incorrect response was that they did not know the legal limit (38.4\% for drink driving and $39.4 \%$ for drunk driving). Of those who nominated a figure for drink driving, overestimation
(18.2\%) was more common than underestimation (15.2\%), while this was reversed for drunk driving, where $14.2 \%$ underestimated the legal limit and 3\% overestimated it. Almost all offenders had no knowledge of how to keep their BAC under the legal limit. Correct answers for the amount of alcoholic beverages that could be consumed to remain under the legal limit were given by only $2 \%$ of participants for spirits, $6.9 \%$ for wine, and $1 \%$ for beer. 


\section{Drinking, Driving, and Exposure to Police Enforcement}

The age when alcohol was first consumed ranged from 830 years (Mean $=19.5$ years; $S D=4.1$ ). Less than half reported that they had previously consumed enough alcohol to put them over the legal limit and had subsequently driven a car $(41.6 \%)$, ridden a motorcycle $(47.5 \%)$, driven another vehicle $(13.9 \%)$, or ridden a bicycle $(34.7 \%)$. In the previous 12 months, 35.7\% reported knowingly being a passenger of a drunk driver. The majority $(77.7 \%)$ reported that at least occasionally they had tried to control the amount of alcohol they had consumed if they were driving. This might be more difficult for Chinese drivers to do than drivers in those countries where information about "standard drinks" equivalence is displayed on alcohol products (e.g., Australia). In relation to levels of exposure to police enforcement, participants were asked how many times they had been stopped by police conducting random breath testing (RBT) in the previous 12 months. Of the entire sample, $72.3 \%$ reported having been stopped once, $18.8 \%$ reported having been stopped twice, with the remaining $8.9 \%$ reporting having been tested on more than two occasions in that one-year period. The majority (77.2\%) admitted their BAC reading was above the legal limit at the time of last testing and $8.9 \%$ did not admit or possibly recognize that their BAC was over the limit. Interestingly, 5.9\% reported not knowing if their BAC reading was above the legal limit when last tested by traffic-police officers conducting RBT, and $7.9 \%$ indicated that they had never been tested. Importantly, about one fifth of the sample $(21.8 \%)$ reported having been previously jailed for drunk driving (i.e., recidivists).

\section{Alcohol Use (AUDIT)}

The mean AUDIT score of these convicted drunk-driving offenders was $11.1(S D=5.9)$, representing a medium level of alcohol problems. Table 1 gives the AUDIT score distribution and shows that more than half of the participants $(66 \%$, or $45 \%+7 \%+4 \%)$ reported medium or even high problematic alcohol use.

There was no significant difference between the mean AUDIT score for females (11.6) and males (11.1), and there was a moderate negative correlation between age and AUDIT score, $r=-0.3, n=101, p<0.01$, representing more problematic drinking among younger offenders.

There were no significant differences in AUDIT scores according to income category. AUDIT scores did not vary significantly according to level of education, with all means lying between 10.2 and 12.9 (see Table 1).

\section{Driving Experience and Alcohol Use}

Offenders were divided into four license groups: novice drivers (licensed for $<2$ years); licensed 2-5 years; licensed 6-10 years; and experienced (licensed 11+ years). The correlation between licensed years and AUDIT scores was $r=-0.63, n=96$, indicating a significant negative correlation between the two variables, $p<0.001$. From
Table 1, it can be seen that a one-way ANOVA showed that the AUDIT score of the novice driver group was significantly higher than the other three groups $(F(3,91)=$ $6.5, p<0.01$ ), with no differences between the other groups.

There was a moderate negative correlation between the age the respondent started drinking and his or her AUDIT score, $r=-0.4, n=100, p<0.01$, such that the younger a participant was when he or she started drinking alcohol, the higher his or her level of alcohol-use disorders. Those who correctly reported knowing the legal BAC limit for drunk driving $(M=9.8, S D=5.16)$ had a significantly lower mean AUDIT score than those who did not $(M=12.2, S D$ $=6.26 ; n=96, t=1.987, p=0.05$ ). Recidivist drunkdriving offenders who had previously been jailed had a higher AUDIT score $(M=15.1, S D=6.48)$ than first-time convicted offenders $(M=9.9, S D=4.9 ; n=95, t=4.075, p$ $<0.001)$.

A multiple regression analysis was used to explore the relationship between AUDIT score and age, gender, years licensed, age at which participants started drinking, marital status, monthly income, education level, knowledge of law and two legal BAC levels, and recidivist status. All predictors were entered simultaneously. The results showed that there was a weak relationship between the age at which a person started drinking alcohol and their AUDIT score $B(1,93)=-0.3016, p<0.05$. In addition, there was a significant difference in AUDIT score, $B(1,95)=4.826, p$ $<0.001$, between recidivist offenders and first-time convicted offenders. There were no other significant relationships identified in the multiple regression analysis (see Table 2).

\section{Discussion}

The current study explored whether convicted drunk drivers in Guangzhou, China, were aware of amended alcohol-related driving legislation and related BAC limits, and whether this knowledge, exposure to enforcement, and the existence of alcohol-use disorders were associated with drunk driving. The majority of offenders were men in their 30s, and approximately two thirds reported medium to high problematic alcohol use, as assessed by the AUDIT. A substantial proportion of the offender sample reported knowing that legislation had recently been amended to make drunk driving (BAC greater than $80 \mathrm{mg} / 100 \mathrm{ml}$ ) a criminal offense. However, their knowledge about legal BAC limits for the two offenses of drink driving (28.3\%) and drunk driving (41.4\%) was surprisingly low, with most of those who did not know the correct limits stating that they did not know, rather than nominating an incorrect figure. Knowledge about how to consume safe levels of alcohol to remain below the BAC levels was lacking, and those who could be described as novice drivers (i.e., licensed for less than 2 years) reported significantly greater alcohol misuse than all others in the sample. 
Table 2

Results of multiple regression Analysis between alcohol use disorders identification test score and predictor variables

\begin{tabular}{|c|c|c|c|c|c|}
\hline \multirow[b]{2}{*}{ Variable } & \multirow[b]{2}{*}{$\boldsymbol{B}$} & \multicolumn{2}{|c|}{ 95\% Confidence Interval } & \multirow[b]{2}{*}{$P$ value } & \multirow{2}{*}{$\begin{array}{l}\text { Partial Eta } \\
\text { Squared }\end{array}$} \\
\hline & & Lower Bound & Upper Bound & & \\
\hline Age & -0.092 & -0.249 & 0.095 & 0.916 & 1.314 \\
\hline Gender (Female as reference) & 0.680 & -2.926 & 4.286 & 0.712 & 0.137 \\
\hline Years licensed, & -0.038 & -0.237 & 0.161 & 0.706 & 0.142 \\
\hline Age commenced drinking & -0.301 & -0.566 & -0.037 & $0.025^{*}$ & 5.000 \\
\hline $\begin{array}{l}\text { Marital status Married (single, widow, } \\
\text { and divorce as reference) }\end{array}$ & -1.112 & -3.424 & 1.2 & 0.346 & 0.889 \\
\hline \multicolumn{6}{|l|}{ Monthly Income (RMB) } \\
\hline 0-2000 & 0.204 & -3.184 & 3.592 & 0.906 & 0.014 \\
\hline $2001-4000$ & -0.069 & -3.362 & 3.224 & 0.967 & 0.002 \\
\hline $4001-6000$ & 0.272 & -4.161 & 4.704 & 0.904 & 0.014 \\
\hline $6001-8000$ & -0.521 & -5.022 & 3.980 & 0.821 & 0.051 \\
\hline $8001-10,000$ & 0.797 & -4.252 & 5.845 & 0.757 & 0.096 \\
\hline More than 10,000 & As reference & & & & \\
\hline \multicolumn{6}{|l|}{ Education level } \\
\hline Primary school & -1.005 & -5.251 & 3.241 & 0.643 & 0.215 \\
\hline Middle school & 0.154 & -2.918 & 3.226 & 0.922 & 0.010 \\
\hline High school and middle college & -0.750 & -3.342 & 1.842 & 0.571 & 0.322 \\
\hline Bachelor degree and higher & As reference & & & & \\
\hline $\begin{array}{l}\text { Know drunk driving amended Criminal } \\
\text { offense (don’t know as reference) }\end{array}$ & 0.266 & -2.795 & 3.327 & 0.865 & 0.029 \\
\hline $\begin{array}{l}\text { Have knowledge of Blood Alcohol } \\
\text { Concentration for Drink driving (don't } \\
\text { have as reference) }\end{array}$ & -1.590 & -4.245 & 1.064 & 0.240 & 1.379 \\
\hline $\begin{array}{l}\text { Have knowledge of BAC for Drunk } \\
\text { driving (don’t have as reference) }\end{array}$ & -1.047 & -3.550 & 1.455 & 0.412 & 0.673 \\
\hline $\begin{array}{l}\text { Recidivist (first convicted offense as } \\
\text { reference) }\end{array}$ & 4.826 & 1.924 & 7.728 & $0.001^{* *}$ & 10.622 \\
\hline
\end{tabular}

$* p<0.05 ; * * p<0.001$

Finally, multiple regression analysis of a range of demographic and exposure variables found that only the age at which someone started drinking had a significant relationship with alcohol misuse, such that those who reported starting to drink at a younger age also reported greater alcohol problems.

Consistent with other research that has suggested that driver characteristics contribute to $95 \%$ of crashes (Sabey \& Taylor, 1980) and that age and gender are key factors for consideration (Miaou \& Lum, 1993), particularly with regard to alcohol-related crashes (Baum, 2000; Eensoo, Paaver, Harro, \& Harro, 2005; Yuan, Li, \& Zhang, 2013), the current study found that most of the drunk-driving offender sample were male with an average age of 33.6 years. Only $9.9 \%$ were women. Previous research on drink driving conducted in Guangxi Province, China, found that of 145 drivers who were involved in alcohol-related crashes, $99 \%$ were male with a mean age of 33.9 years (SD = 9.2) (Yuan et al., 2013). Similarly, research conducted in Australia (e.g., Baum, 2000; Eensoo et al., 2005) typically has found that there is a higher incidence of drinking and drink driving among males than females. Together, these findings suggest that the target population for further training, education, and interventions for prevention of alcohol-related driving in China should be younger males.

Results from the current study indicate that alcohol problems among this sample of drunk-driving offenders were more serious than among a general car-driver cohort recruited in the same city at the same time period (see Jia et al., 2013). When comparing AUDIT scores of the general sample $(M=7.4, S D=5.4)$ (Jia et al., 2013) with the current offender sample $(M=11.1, S D=5.9)$, the offenders exhibited significantly greater severity of alcohol-use disorders. Offenders exhibited a medium level of alcoholuse disorders (i.e., AUDIT scores between 8-15.9), and it is worth noting that $20 \%$ were classified as high or in need of further diagnostic evaluation for alcohol dependence. Interestingly, offenders also had more accurate knowledge about drink-driving and drunk-driving BAC legal levels than the general sample. This difference is perhaps not 
surprising and possibly reflects the nature of the current sample (i.e., drunk-driving offenders who have recently been convicted). In other words, after they were apprehended, their exposure to legal proceedings might have provided the opportunity to learn about the two legal BACs, although it is acknowledged that this is only one possible explanation for this finding. Importantly, however, even though a reasonable number of offenders knew the correct BAC levels, there was little knowledge about how to remain under the legal BAC limit when drinking. It is clear that further education on this issue is needed in Guangzhou.

In the current study, there was no significant bivariate relationship between monthly income level and frequency of drinking. In 2011, the average monthly income was 4,800 RMB for Guangzhou registered employees. In the Chinese alcohol beverage market, the prices of bottles of beer, wine, and spirits vary from around a few RMB to hundreds or thousands of RMB. This spread of prices allows for ready substitution to maintain alcohol consumption without spending more money, although this is a speculative suggestion. In western countries, there have been conflicting findings regarding the relationship between income levels and drunk-driving problems. For example, research from Australia and Europe has found that drunk driving is associated with lower income (Baum, 2000; Golias \& Karlaftis, 2001); however, research conducted in the United States has not (Gruenewald \& Mitchell, 1996; Wilson \& Jonah, 1985). A larger sample is needed to further explore the socio-economic factors related to drinking and driving behaviors in China.

The majority of offenders in the current sample were aware that drunk driving became a criminal offense in May 2011, but this would be expected, given their recent conviction, subsequent to that change. As noted above, however, their knowledge about legal BAC limits for drink-driving and drunk-driving offenses was surprisingly low, even for those who knew about the new law. By comparison, the Global Road Safety Partnership (GRSP) conducted a baseline survey on drink driving in two other Chinese cities in 2007 and found that only $4.8 \%$ of drivers were aware of the lower limit, which suggests an improvement in knowledge about BAC levels over time (Yuan et al., 2013). However, it is worth noting that the GRSP research was conducted prior to legislative changes in 2011, and therefore public awareness of legal drinking levels at that time could reasonably be expected to be lower than after 2011 .

The finding that the more recently licensed drunk-driving offenders in the current sample (defined as novice drivers) had higher AUDIT scores is arguably consistent with the rapid increases in alcohol consumption and related problems that have been observed in China (Hao, Su, \& Chen, 2007; Zhang, Zhao, \& Zhou, 2004). As noted above, further analysis suggested that this relationship was explained by the age when offenders first began drinking rather than license status; however, it raises some issues regarding novice drivers that merit further investigation. Novice driver training is mandated in China and involves off-road training focused largely on vehicle handling skills and classroom learning (Senserrick, Yu, Boufous, Stevenson, \& Ivers, 2012). However, it is currently unclear how much education regarding the risks of alcohol-related driving and legal BAC levels is taught. Furthermore, unlike many countries, China's novice drivers are not subject to regulations shown to have been protective, such as a zero BAC requirement at all times (Senserrick, Yu, Wei, Stevenson, \& Ivers, 2011). The findings of the current study suggest that there is scope to examine and potentially improve the way in which novice driver education and management is conducted in China, especially with regard to the risks of drinking and driving.

The proportion (56\%) of medium- or higher-level alcohol problems in drunk-driving offenders in this Guangzhou sample was higher than other studies conducted in Chinese societies. For instance, an epidemiological study of 3,171 residents in Lhasa, Tibet, found that $16.2 \%$ had alcohol-use disorders (cut-off point 8) (Guo et al., 2008). Another study in Hong Kong reported that a higher percentage of respondents from hospitals (14.5\%) had alcohol problems compared to those from the community (6.2\%) or university (5.3\%) (cut-off point 8) (Leung \& Arthur, 2000). In addition, recidivist drink- or drunk-driving offenders had higher AUDIT scores than first-time offenders in the current study. This is consistent with findings from Australian research where the majority of a group of recidivist drink drivers did not report being motivated to reduce their alcohol consumption in spite of being sanctioned for a drinking-related offense (Freeman et al., 2005). For such offenders, their drinking behavior may be heavily entrenched and can prove difficult to change (Freeman, Schonfeld, \& Sheehan, 2007).

Multiple regression analysis showed that once all variables were considered together, the length of time that someone had been licensed was no longer significantly associated with level of alcohol misuse. One of two significant relationships (weak yet significant) was between the age when offenders started drinking and their level of alcohol problems. This is consistent with Hingson, Heeren, Levenson, Jamanka, and Voas (2002), who found that the earlier the age respondents started drinking, the more likely they were to report driving after drinking too much and being in a crash. Our finding might, in part, reflect a change in alcohol consumption patterns in China over time and may be clarified by a study with a larger sample size. In China, it is illegal to sell alcohol to a minor (under 18 years of age) according to the 19th and 30th Articles in the Regulation of Applications for Sale of Alcohol Licenses (The Department of Commerce of the People's Republic of China, 2006). There are no minimum legal drinking age laws that specify an age below which people cannot purchase or publicly consume alcohol in China, so this should be considered as a possible intervention, especially since the youngest offender in our sample reported to be 17 years old. The other significant relationship found was that recidivist offenders had a higher AUDIT score than firsttime convicted offenders. Considering the high rate of recidivism in the sample $(21.8 \%)$ and serious alcohol misuse problems for recidivist offenders, rehabilitation programs should be considered. 
This research represents the first investigation of drunkdriving offenders in China. Despite its important contribution to the knowledge in this area, several limitations are acknowledged. Firstly, the sampling method chosen to gain access to participants in detention meant that the research team could not directly interact with participants. Instead, uniformed police officers disseminated and collected questionnaires from offenders, which may have resulted in biased, incomplete, or inaccurate responses-for example, because participants thought that the police would look at their responses, they may have modified them to create a favorable impression. Additionally, the use of self-report measures alone, without objective data to confirm prior offenses, may limit the validity of our conclusions. Despite these limitations, it is encouraging that our findings are reasonably consistent with others in the broader literature relating to alcoholrelated driving offenders and alcohol use among recidivists.

Overall, these results suggest the need to better promote safe levels of drinking and also the need to encourage those with alcohol-use problems to separate their drinking and driving in the future. A variety of strategies could be considered by Chinese authorities to assist in this regard, including public education campaigns to raise awareness of measures of alcohol consumption, including standard drinks, and of the risks of drink and drunk driving; introduction of drink- and drunk-driver rehabilitation programs; and/or the installation of alcohol ignition interlocks in vehicles to assist in separating drinking from driving.

\section{Acknowledgements}

The authors gratefully acknowledge the assistance of staff from Guangdong Institute of Public Health, Guangdong Centre for Disease Control and Prevention, and police officers from the jail authority.

\section{References}

Australian Drug Foundation. (2002). Driving for life: drink driver education manual. Sydney, Australia: Artways.

Babor, T., \& Grant, M. (1989). From clinical research to secondary prevention: International collaboration in the development of the Alcohol Use Disorders Identification test (AUDIT). Alcohol Health \& Research World, 13, 371-374.

Baum, S. (2000). Drink driving as a social problem: Comparing the attitudes and knowledge of drink driving offenders and the general community. Accident Analysis and Prevention, 32(5), 689-694. doi:0.1016/S0001-4575(99)00106-2

Cochrane, J., Chen, H. H., Conigrave, K. M., \& Hao, W. (2003). Alcohol use in China. Alcohol and Alcoholism, 38, 537-542.

Eensoo, D., Paaver, M., Harro, M., \& Harro, J. (2005). Predicting drunk driving: Contribution of alcohol use and related problems traffic behaviour, personality and platelet monoamine oxidase (MAO) activity. Alcohol and Alcoholism, 40(2), 140-146.

Freeman, J. E., Liossis, P., Schonfeld, C. C., Sheehan, M. C., Siskind, V., \& Watson, B. C. (2005). Self-reported motivations to change and self-efficacy levels for a group of recidivist drink drivers. Addictive Behaviors, 30(6), 1230-1235.

Freeman, J. E., Schonfeld, C. C., \& Sheehan, M. C. (2007). Reoffending after program completion: A study into the characteristics of the hardcore recidivist drink driver. In: International Conference on Alcohol Drugs and Traffic Safety T2007, 26-30 August 2007, Seattle, United States.

Golias, I., \& Karlaftis, M. G. (2001). An international comparative study of self-reported driver behavior. Transportation Research Part F: Traffic Psychology and Behaviour, 4(4), 243-256. doi:10.1016/S13698478(01)00026-2

Gruenewald, P. J., \& Mitchell, P. R. (1996). Drinking and driving: Drinking patterns and drinking problems. Addiction, 91(11), 1637-1649.

Guangdong Statistics Bureau. (2012). The status of annual average income in Guangzhou in 2011. Retrieved from http://www.gzstats.gov.cn/tjgb/qtgb/201206/ t20120629_29123.htm

Guo, W., Lanzi, G., Luobu, O., Ma, X., Zhen, P., Ji, Y., ... Li, T. (2008). An epidemiological survey of alcohol use disorders in a Tibetan population. Psychiatry Research, 159(1-2), 56-66. doi:10.1016/j.psychres. 2007.09.012

Hao, W., Su, Z., \& Chen, H. (2007). Drinking and drinking-related problems in China. Chinese Journal of Drug Dependence, 42, 595-601.

Hingson, R., Heeren, T., Levenson, S., Jamanka, A., \& Voas, R. (2002). Age of drinking onset, driving after drinking, and involvement in alcohol related motorvehicle crashes. Accident Analysis \& Prevention, 34(1), 85-92. doi:10.1016/S0001-4575(01)00002-1

Jia, K., King, M., Sheehan, M., Fleiter, J., Ma, J., \& Zhang, J. (2013). Baseline study of alcohol dependence among general drivers and drunk driving offenders in Guangzhou, China. Proceedings from the Road Safety Research, Policing and Education Conference 2013. Retrieved from http://acrs.org.au/files/arsrpe/ Paper\%20187\%20-\%20Jia\%20\%20Alcohol\%20and\%20Driving.pdf

Kong, B. (2012, May 7). The rate of drunk driving decreased $70 \%$ at the punishment anniversary: Dispute and expectation. Retrieved from http://news.xatrm.com/gov/press/zyrmzfwxw/BF1FAF C97D2D722BE040007F01006DE3.html

Leung, S. F., \& Arthur, D. (2000). The alcohol use disorders identification test (AUDIT): Validation of an instrument for enhancing nursing practice in Hong Kong. International Journal of Nursing Studies, 37(1), 57-64. doi:10.1016/s0020-7489(99)00052-8

Li, Y., Xie, D., Nie, G., \& Zhang, J. (2012). The drink driving situation in China. Traffic Injury Prevention, 13(2), 101-108.

Ma, J. (2012). Comparison and analysis of the economic development between Guangdong province with the whole nation. Guangzhou: Statistics Bureau of 
Guangdong Province. Retrieved from http://www.gdstats.gov.cn/tjzl/tjfx/201306/t20130617 _122574.html

Miaou, S. P., \& Lum, H. (1993). Modeling vehicle accidents and highway geometric design relationships. Accident Analysis and Prevention, 25, 689-709.

Sabey, B. E., \& Taylor, H. (1980). The known risks we run: The highway. Report SR567. Crowthorne, England: Transport Research Laboratory.

Senserrick, T., Yu, X., Wei, D., Stevenson, M., \& Ivers, R. (2011). Development of a supplementary education and training program for novice drivers in China. Journal of the Australian College of Road Safety, 22(2), 36-41.

Senserrick, T., Yu, J., Boufous, S., Stevenson, M., \& Ivers, R. (2012). Research challenges and findings from a driver training pilot study in China. Paper presented at the Australasian Road Safety Research, Policing and Education Conference 2012, Wellington, New Zealand.

Ministry of Commerce of the People's Republic of China. (2006). Regulation of application for sale alcohol licence. Beijing, China.

Traffic Management Bureau under Ministry of Public Security. (2012). Annual Report of Road Traffic Crashes in People's Republic of China (in Chinese). Beijing, China.

Tran, N. T., Bachani, A. M., Pham, V. C., Lunnen, J. C., Jo, Y., Passmore, J., \& Hyder, A. A. (2011). Drinking and driving in Vietnam: Public knowledge, attitudes, and practices. Traffic Injury Prevention, 13(Suppl. 1), 37-43. doi:10.1080/15389588.2011.636779

Watson, B., \& Freeman, J. (2007). Perceptions and experiences of random breath testing in Queensland and the self-reported deterrent impact on drinkdriving. Traffic Injury Prevention, 8, 11-19. doi:10.1080/15389580601027360

Wen, X. (2012, September 12). Guangzhou traffic police officers enter club to publicise the harm of drink driving. Retrieved from http://www.gdga.gov.cn/jwzx/ gdjx/gzsj/201209/t20120912_617101.html

Wilson, R. J., \& Jonah, B. A. (1985). Identifying impaired drivers among the general driving population. Journal of Studies on Alcohol and Drugs, 46(6), 531-537.

Yuan, A., Li, Y., \& Zhang, J. (2013). The result of a baseline survey on drink driving in Nanning and Liuzhou of Guangxi Province, China. Traffic Injury Prevention, 14(3), 230-236.

Zhang, G., Yau, K. W. K., \& Chen., G. (2013). Risk factors associated with traffic violations and accident severity in China. Accident Analysis and Prevention, 59C, 1825.

Zhang, L. F., Zhao, L. C., \& Zhou, B. F. (2004). Alcohol consumption and incidence of ischemic stroke in male Chinese. Chinese Journal of Epidemiology, 25, 954957. 